PROCEEDINGS OF THE

AMERICAN MATHEMATICAL SOCIETY

Volume 138, Number 8, August 2010, Pages 2701-2714

S 0002-9939(10)10307-4

Article electronically published on April 5, 2010

\title{
COMPRESSION BOUNDS FOR WREATH PRODUCTS
}

\author{
SEAN LI \\ (Communicated by Alexander N. Dranishnikov)
}

\begin{abstract}
We show that if $G$ and $H$ are finitely generated groups whose Hilbert compression exponent is positive, then so is the Hilbert compression exponent of the wreath product $G\{H$. We also prove an analogous result for coarse embeddings of wreath products. In the special case $G=\mathbb{Z}, H=\mathbb{Z} \imath \mathbb{Z}$ our result implies that the Hilbert compression exponent of $\mathbb{Z} \imath(\mathbb{Z} \imath \mathbb{Z})$ is at least $1 / 4$, answering a question posed by several authors.
\end{abstract}

\section{INTRODUCTION}

Given two groups, $G$ and $H$, we denote their wreath product $G$ \& $H$ to be the group of all pairs $(f, x)$, where $f: H \rightarrow G$ is finitely supported (i.e. $f(z)=e_{G}$ for all but finitely many $z \in H)$ and $x \in H$. We will let $\operatorname{supp} f$ denote $\left\{x \in H: f(x) \neq e_{G}\right\}$. Let $T_{x} g(z)=g\left(x^{-1} z\right)$. The product is given by

$$
(f, x) \cdot(g, y)=\left(z \mapsto f(z) g\left(x^{-1} z\right), x y\right)=:\left(f T_{x} g, x y\right) .
$$

Using the usual heuristic of interpreting wreath product word metrics as lamplighter walks (see e.g. NP08), we can interpret the metric as a traveling salesman tour on $H$. For this paper, all groups will be assumed to be infinite unless specified otherwise. If $S$ and $T$ generate $G$ and $H$, respectively, then $G$ ? $H$ is generated by the set $\left\{\left(e_{G^{H}}, t\right): t \in T\right\} \cup\left\{\left(\delta_{s}, e_{H}\right): s \in S\right\}$, where $\delta_{s}$ is the function taking the value $s$ at $e_{H}$ and $e_{G}$ everywhere else. We will denote $\mathcal{L}_{G}(H)$ to be the wreath product where the generating set of $G$ is taken to be all elements.

Note that mappings $f$ of a metric space into $L_{1}$ induce pseudometrics given by

$$
d_{f}(x, y)=\|f(x)-f(y)\|_{1} .
$$

A cut in $G$ is a subset $A$ of $G$. We say a cut $A$ separates a subset $B$ (denoted $A \vdash B$ ) if $B$ intersects both $A$ and $A^{c}$ nontrivially. Given a measure $\mu$ on $\mathcal{C}$, a subset of the set of cuts of $G$, we can define a cut pseudometric by

$$
d_{\mu}(x, y)=\int_{\mathcal{C}}\left|\mathbf{1}_{E}(x)-\mathbf{1}_{E}(y)\right| d \mu(E) .
$$

It is known that pseudometrics induced from embeddings into $L_{1}$ come from cut pseudometrics and vice versa DL97, CK06.

Received by the editors September 2, 2009, and, in revised form, December 3, 2009.

2010 Mathematics Subject Classification. Primary 20F65, 51F99.

This work was supported in part by NSF grants CCF-0635078 and CCF-0832795.

(C)2010 American Mathematical Society 
For metric spaces $X$ and $Y$, we say that $f: X \rightarrow Y$ is a coarse embedding if there exist increasing unbounded functions $\rho_{1}, \rho_{2}: \mathbb{R}_{+} \rightarrow \mathbb{R}_{+}$satisfying the inequality

$$
\rho_{1}\left(d_{X}(x, y)\right) \leq d_{Y}(f(x), f(y)) \leq \rho_{2}\left(d_{X}(x, y)\right) .
$$

In GK04, Guentner and Kaminker gave a simple and elegant way of further strengthening coarse embeddability. Let $G$ be a finitely generated group and $d$ the word metric with respect to its finite set of generators. The $L_{p}$ compression exponent of $G$, which we denote by $\alpha_{p}^{*}(G)$, is the supremal $\alpha$ for which there exists a Lipschitz map $f: G \rightarrow L_{p}$ satisfying the inequality

$$
\|f(x)-f(y)\|_{p} \gtrsim d(x, y)^{\alpha} .
$$

We will use $\lesssim$ and $\gtrsim$ to denote inequality up to multiplication by some nonzero constant. If $x \lesssim y$ and $y \lesssim x$, then we will say $x \asymp y$. As all word metrics of a group with respect to finite generators are bi-Lipschitz equivalent, we see that the $L_{p}$ compression exponent of a group is in fact an algebraic invariant.

Furthermore, a map $f: G \rightarrow X$ where $X$ is a Banach space is called $G$ equivariant if it is given by the orbit of a vector $v \in X$ under the action of $G$ on $X$ by affine isometries. That is, there is a representation $\alpha: G \rightarrow \operatorname{Isom}(X)$ such that $f(g)=\alpha(g) \cdot 0$. Note that the pseudometric $\|f(g)-f(h)\|_{X}$ is then $G$-invariant. A group is said to have the Haagerup property if there exists an equivariant function $f: G \rightarrow L_{2}$ such that $\inf \left\{\|f(g)-f(h)\|_{2}: d_{G}(g, h) \geq t\right\}$ tends to infinity with $t$. We will say that this equivariant mapping is metrically proper.

We refer to the book $\mathrm{CCJ}^{+} 01$ for more information on the Haagerup property, where in particular there is a discussion of which group operations preserve this property. General semidirect products do not necessarily preserve the Haagerup property, or even the property of having a positive compression exponent, as shown by the example $\mathbb{Z}^{2} \rtimes \mathrm{SL}_{2}(\mathbb{Z})$. Nevertheless, a recent breakthrough of de Cornulier, Stalder, and Valette dCSV09 shows that wreath products do preserve the Haagerup property.

In this paper we show that the property of having a positive compression exponent is also preserved under wreath products. Our approach uses crucially a tool from dCSV09, namely a method for lifting cut pseudometrics from a group $H$ to its associated lamplighter group $\mathcal{L}_{G}(H)$. Several authors previously investigated the behavior of compression exponents under the wreath product operation in various special cases AGS06, ANP09, Gal08, NP08, NP09, SV07, Tes09. All known examples with calculable compression exponent have shown that the wreath product operation preserves positivity of compression exponents, but it was unknown if this was true in general. We prove that this is indeed the case when $p \in[1,2]$.

We first prove that a positive $L_{p}$ compression exponent is preserved for the special case when $p=1$. It is a standard fact that $L_{p}$ isometrically embeds into $L_{1}$ for $p \in(1,2]$ (see e.g. BL00]), so this reduction can always be done. We then lift the cut measure generated by the cut decomposition of the $L_{1}$ embedding of $H$ to the cuts of $G$ ? $H$ using the method of dCSV09. By the correspondence between $L_{1}$ embeddings and cut measures, this gives us an embedding of $G$ ? $H$ into $L_{1}$, which we show has positive $L_{1}$ compression. To get the $L_{p}$ case, we then embed back into $L_{p}$ using a standard technique. This gives us the following theorem. 
Theorem 1.1. For $p \geq 1$, we have:

$$
\alpha_{p}^{*}(G \succ H) \geq \max \left\{\frac{1}{p}, \frac{1}{2}\right\} \cdot \min \left\{\alpha_{1}^{*}(G), \frac{\alpha_{1}^{*}(H)}{1+\alpha_{1}^{*}(H)}\right\} .
$$

It has been previously asked [AGS06, Tes09, NP09] whether $\mathbb{Z} \imath(\mathbb{Z} \imath \mathbb{Z})$ has a positive Hilbert compression exponent. Using the fact that $\alpha_{1}^{*}(\mathbb{Z} \imath \mathbb{Z})=1$ [NP08] we get a lower bound of $\alpha_{2}^{*}(\mathbb{Z} \imath(\mathbb{Z} \imath \mathbb{Z})) \geq 1 / 4$.

Remark 1.1. Write $G_{1}=\mathbb{Z} \imath \mathbb{Z}$ and $G_{k+1}=\mathbb{Z} \imath G_{k}$. Since $G_{1}$ admits a bi-Lipschitz embedding into $L_{1}$ [NP08, it follows from Theorem 1.1 that for all integers $k$ we have $\alpha_{1}^{*}\left(G_{k}\right) \geq \frac{1}{k}$. The question whether or not $\inf _{k \in \mathbb{N}} \alpha_{1}^{*}\left(G_{k}\right)$ is strictly positive remains an interesting open problem.

In dCSV09, the authors showed that coarse embeddability into $L_{p}$ for $p \in[1,2]$ is preserved by wreath products. We use the same construction as in the positive $L_{p}$ compression exponent theorem to give a somewhat simpler constructive proof with quantitative moduli bounds.

Theorem 1.2. If $G$ and $H$ embed coarsely into $L_{1}$, then $G$ i $H$ embeds coarsely into $L_{p}$ for $p \geq 1$. Specifically, suppose we have mappings $\psi: H \rightarrow L_{1}$ and $\phi: G \rightarrow L_{1}$ satisfying the compression bounds

$$
\begin{aligned}
& \rho_{1}(d(x, y)) \leq\|\psi(x)-\psi(y)\|_{1} \leq \rho_{2}(d(x, y)), \\
& \tau_{1}(d(x, y)) \leq\|\phi(x)-\phi(y)\|_{1} \leq \tau_{2}(d(x, y)) .
\end{aligned}
$$

Then there is a mapping $\Phi: G \imath H \rightarrow L_{p}$ satisfying the compression bounds

$$
\nu_{1}(d((f, x),(g, y))) \lesssim\|\Psi(f, x)-\Psi(g, y)\|_{p} \lesssim \nu_{2}(d((f, x),(g, y))),
$$

where

$$
\begin{aligned}
\nu_{2}(t)= & t^{\max \left\{\frac{1}{2}, \frac{1}{p}\right\}}, \\
\nu_{1}(t)= & {\left[\operatorname { m i n } \left\{\sqrt{\min \left\{\sum_{k=1}^{n} \rho_{1}\left(s_{k}\right): s_{k} \geq 0, \sum_{k=1}^{n} s_{k}=t_{1}\right\}}\right.\right.} \\
& \left.\left.+\sum_{j=2}^{m} \tau_{1}\left(t_{j}\right): t_{j} \geq 0, \sum_{j=1}^{m} t_{j}=t\right\}\right]^{\max \left\{\frac{1}{2}, \frac{1}{p}\right\}}
\end{aligned}
$$

In section 3 , we show that $\nu_{1}$ is an increasing unbounded function.

We also show that equivariance is preserved by this construction. Taken with coarse embeddability, we get a somewhat simpler proof of the Haagerup property result of dCSV09,

Theorem 1.3. If $G$ and $H$ have the Haagerup property, then so does $G \imath H$. The compression bounds can be taken to be the same as those in Theorem 1.2.

As a reduction to $L_{1}$ embeddings is part of the proof, we also show that the property of having a metrically proper equivariant map into $L_{1}$ is preserved under wreath products.

Remark 1.2. As mentioned before, this method crucially uses the fact that $L_{p}$ isometrically embeds into $L_{1}$ for $p \in(1,2]$ to show that $L_{p}$ embeddability is preserved by wreath products. For $p>2$, this is no longer true. It is unknown if $G ? H$ 
embeds coarsely into $L_{p}$ for $p>2$ if $G$ and $H$ do so for $p>2$ but not for $p \in[1,2]$. The question of positive compression bounds also remains open, and it would be interesting to develop a method for this parameter range.

\section{Positive $L_{p}$ Compression exponent}

We start by proving the following special case.

Proposition 2.1. $\alpha_{1}^{*}\left(\mathcal{L}_{G}(H)\right) \geq \frac{\alpha_{1}^{*}(H)}{1+\alpha_{1}^{*}(H)}$.

We begin by giving a method of extending measures on cuts of $H$ to measures on cuts of $\mathcal{L}_{G}(H)$. This is a specialization of the method used in dCSV09. Before we start the proof, as a warm-up, we give a description of the cuts for the case $C_{2} \prec \mathbb{Z}^{d}$. Note that the cuts in $\mathbb{Z}^{d}$ are just half-spaces orthogonal to some axis in $\mathbb{Z}^{d}$. The cut measure is just the discrete uniform measure on all the half-spaces. Elements of $C_{2} \prec \mathbb{Z}^{d}$ can be thought of as finite subsets of $\mathbb{Z}^{d}$ with a special point for the initial lamplighter position.

Let $H$ be a half-space. Then, given a finite subset of points $A$ outside $H$, we define the cut $E(H, A)$ to be the collection of all elements of $C_{2}$ 乙 $\mathbb{Z}^{d}$ whose initial lamplighter position is inside $H$ and whose subset outside of $H$ agrees with $A$. The total collection of cuts on $C_{2} \prec \mathbb{Z}^{d}$ is simply the collection of all such cuts for all $H$ and all finite subsets outside of each $H$. The cut measure is simply the discrete uniform measure on all the possible cuts.

We now give the full proof of Proposition 2.1.

Proof. As $\alpha_{1}^{*}(H)=\alpha$, for every $\varepsilon \in(0, \alpha)$, we can find a Lipschitz function $\phi$ : $H \rightarrow L_{1}$ so that for every $x, y \in H$, we have

$$
\|\phi(x)-\phi(y)\|_{1} \gtrsim d_{H}(x, y)^{\alpha-\varepsilon} .
$$

Let $\rho$ be the cut measure on $\mathcal{C}$ induced by the cut decomposition of $\phi$. We then know that $\rho$ induces a cut metric. We can complete $\mathcal{C}$ under taking complements and then define a new cut measure $\mu(A)=\rho(A)+\rho(\check{A})$, where

$$
\check{A}=\left\{B^{c}: B \in A\right\} .
$$

The $\sigma$-algebra of the completion can simply be taken as the $\sigma$-algebra generated by $\mathcal{C}$ and $\check{\mathcal{C}}$. We will still denote this by $\mathcal{C}$. Note then that $\mu$ is invariant under complements and differs from $\rho$ by at most a multiplicative factor of 2. Given a cut $B$ and finitely supported functions $f: B^{c} \rightarrow G$, we let

$$
E(B, f)=\left\{(g, x) \in \mathcal{L}_{G}(H): x \in B,\left.g\right|_{B^{c}}=f\right\} .
$$

We will abuse notation and write $E(B, h)$ for $h: H \rightarrow G$ to mean the obvious cut using the restriction of $h$. Note that even if there exists an element $y \in B$ such that $h(y) \neq h^{\prime}(y)$, we still have that $E(B, h)=E\left(B, h^{\prime}\right)$ if $\left.h\right|_{B^{c}}=\left.h^{\prime}\right|_{B^{c}}$.

We can construct a measure $\nu$ on the set of cuts given by

$$
\mathcal{H}=\bigcup_{\substack{B \in \mathcal{C} \\ \text { supp } f<\infty}} \bigcup_{\substack{f: B^{c} \rightarrow G \\ \text { sups }}}\{E(B, f)\},
$$

which induces a cut metric on $\mathcal{L}_{G}(H)$. Indeed, consider the space

$$
\tilde{\mathcal{C}}=\bigsqcup_{\substack{f: H \rightarrow G \\ \sup f<<\infty}}\{(B, f): B \in \mathcal{C}\}
$$


Note that $\tilde{\mathcal{C}}$ is a countable union of sets bijective to $\mathcal{C}$. Indeed, $H$ is countable and the set of finite subsets of $H$ is thus also countable. Define the $\sigma$-algebra on $\tilde{\mathcal{C}}$ to be the $\sigma$-algebra generated by the $\sigma$-algebra of each of the factors. There is an obvious measure $\tilde{\mu}$ on $\tilde{\mathcal{C}}$ that restricts to $\mu$ on each of the factors. That is, for every $f: H \rightarrow G$ and $A \subset \mathcal{C}$, we have that $\tilde{\mu}(\{(B, f): B \in A\})=\mu(A)$.

Define the injection $\iota: \mathcal{H} \hookrightarrow \tilde{\mathcal{C}}$ given by $\iota(E(B, f))=(B, f)$, where $\left.f\right|_{B^{c}}=f$ and $\left.f\right|_{B}=e_{G^{B}}$. We can define a pullback $\sigma$-algebra on $\mathcal{H}$ so that $\iota$ is measurable. We then define the measure $\nu$ on $\mathcal{H}$ by $\nu(A)=\tilde{\mu}(\iota(A))$. This is well defined as $\mathcal{H}$ has the pullback $\sigma$-algebra of $\iota$. As $\iota$ is injective and $\mu$ is countably additive, $\nu$ is countably additive and thus indeed a measure.

One can see that

$$
\begin{aligned}
d_{\nu}((f, x),(g, y)) & =\nu(\{B \in \mathcal{H}: B \vdash\{(f, x),(g, y)\}\}) \\
& \asymp \mu\left(\left\{B \in \mathcal{C}: B \vdash\left(\operatorname{supp} f^{-1} g\right) \cup\{x, y\}\right\}\right) .
\end{aligned}
$$

This follows from easy case analyses. In the $\gtrsim$ direction, suppose that $B \vdash$ $\left(\operatorname{supp} f^{-1} g\right) \cup\{x, y\}$ (recall that we say $B$ separates $A$ or $B \vdash A$ if $A \cap B \neq \emptyset$ and $\left.A \cap B^{c} \neq \emptyset\right)$. If $B \vdash\{x, y\}$, then from a case analysis of whether $x$ or $y$ is in $B$, either $E(B, f)$ or $E(B, g)$ separate $\{(f, x),(g, y)\}$. No other possible cut can separate $\{(f, x),(g, y)\}$ using the same cut $B$. Indeed if $E(B, h)$ is some other cut, then the restriction of $f$ and $g$ outside $B$ could not be equal to $h$ and so $(f, x)$ and $(g, y)$ would both not be in $E(B, h)$. If $\{x, y\} \subset B$, then $E(B, f)$ and $E(B, g)$ both separate as there must be an element of $\operatorname{supp} f^{-1} g$ outside of $B$. As before, no other $E(B, h)$ can separate. If $\{x, y\} \cap B=\emptyset$, then $E\left(B^{c}, f\right)$ and $E\left(B^{c}, g\right)$ are the cuts that separate as $B$ contains an element of $\operatorname{supp} f^{-1} g$. As $\mathcal{C}$ is closed under taking complements, we have that these cuts are in $\mathcal{H}$.

In the $\lesssim$ direction, suppose $E(B, h)$ separates $\{(f, x),(g, y)\}$. Note that $x$ and $y$ cannot both be in $B^{c}$ as then $(f, x)$ and $(g, y)$ are not in $E(B, h)$. If $B \vdash\{x, y\}$, then we are done. Suppose then that $x, y \in B$. Then we must have that $\left.g\right|_{B^{c}} \neq\left. f\right|_{B^{c}}$ and so $\left(\operatorname{supp} f^{-1} g\right) \cap B^{c} \neq \emptyset$.

In particular,

$$
d_{\nu}((f, x),(g, y)) \gtrsim \max \left\{d_{\mu}(x, y) \cup\left\{d_{\mu}(x, z)\right\}_{z \in \operatorname{supp} f^{-1} g}\right\} .
$$

Thus, we have a function $F: \mathcal{L}_{G}(H) \rightarrow L_{1}$ such that

$$
\|F(f, x)-F(g, y)\|_{1}=d_{\nu}((f, x),(g, y)) \gtrsim \max \left\{d_{\mu}(x, y) \cup\left\{d_{\mu}(x, z)\right\}_{z \in \operatorname{supp} f^{-1} g}\right\} .
$$

This function is Lipschitz. By the triangle inequality and the traveling salesman interpretation of the wreath product word metric, it suffices to check this for the cases when $(f, x)$ and $(g, y)$ differ by only a generator. If $(f, x)$ and $(g, x)$ differ by an element of the form $\left(\delta_{g}, e_{H}\right)$ for $g \in G$, then $F(f, x)=F(g, x)$. Indeed, $\operatorname{supp} f^{-1} g \cup\{x, x\}=\{x\}$, and there are no cuts that can separate a singleton. Suppose then that $(f, x)$ and $(f, y)$ differ by $(0, t)$, where $t$ is a generator of $H$. Then $\operatorname{supp} f^{-1} f \cup\{x, y\}=\{x, y\}$ and by the above,

$$
\|F(f, x)-F(f, y)\| \lesssim \mu(\{B \in \mathcal{C}: B \vdash\{x, y\}\})=d_{\mu}(x, y) \lesssim d((f, x),(f, y)) .
$$

By our choice of $\mu$, we have that

$$
\begin{aligned}
d((f, x),(g, y)) & \gtrsim\|F(f, x)-F(g, y)\|_{1} \\
& \gtrsim \max \left\{d_{H}(x, y)^{\alpha-\varepsilon} \cup\left\{d_{H}(x, z)^{\alpha-\varepsilon}\right\}_{z \in \operatorname{supp} f^{-1} g}\right\} .
\end{aligned}
$$


For a finitely supported function $f: H \rightarrow G$ define $\Lambda(f) \in \ell_{1}(H \times G)$ by

$$
\Lambda(f)_{x y}=\left\{\begin{array}{cl}
\frac{1}{2} & y=f(x) \\
0 & \text { otherwise. }
\end{array}\right.
$$

Thus for $f, g: H \rightarrow G$ we have $\|\Lambda(f)-\Lambda(g)\|_{1}=\left|\operatorname{supp} f^{-1} g\right|$. Now, consider the function

$$
\begin{aligned}
\Psi: \mathcal{L}_{G}(H) & \rightarrow \ell_{1}(H \times G) \oplus L_{1} \\
(f, x) & \mapsto\left(\frac{\alpha-\varepsilon}{1+\alpha-\varepsilon} \Lambda(f)\right) \oplus\left(\frac{1}{1+\alpha-\varepsilon} F(f, x)\right) .
\end{aligned}
$$

This function is clearly Lipschitz. Bounding from below, we get

$$
\begin{aligned}
& \|\Psi(f, x)-\Psi(g, y)\|_{1} \gtrsim \frac{\alpha-\varepsilon}{1+\alpha-\varepsilon}\left|\operatorname{supp} f^{-1} g\right| \\
& \quad+\frac{1}{1+\alpha-\varepsilon} \max \left\{d_{H}(x, y)^{\alpha-\varepsilon} \cup\left\{d_{H}(x, z)^{\alpha-\varepsilon}\right\}_{z \in \operatorname{supp} f^{-1} g}\right\} \\
& \geq \max \left\{\left(\left|\operatorname{supp} f^{-1} g\right|\right.\right. \\
& \left.\left.\quad \cdot \max \left\{d_{H}(x, y) \cup\left\{d_{H}(x, z)\right\}_{z \in \operatorname{supp} f^{-1} g}\right\}\right)^{\frac{\alpha-\varepsilon}{1+\alpha-\varepsilon}}, \frac{d_{H}(x, y)^{\alpha-\varepsilon}}{1+\alpha-\varepsilon}\right\} \\
& \gtrsim\left(\left(1+\left|\operatorname{supp} f^{-1} g\right|\right) \cdot \max \left\{d_{H}(x, y) \cup\left\{d_{H}(x, z)\right\}_{z \in \operatorname{supp} f^{-1} g}\right\}\right)^{\frac{\alpha-\varepsilon}{1+\alpha-\varepsilon}} \\
& \gtrsim d((f, x),(g, y))^{\frac{\alpha-\varepsilon}{1+\alpha-\varepsilon}} .
\end{aligned}
$$

The second inequality above is a consequence of the concavity of the logarithm, and the fourth inequality above comes from the triangle inequality. Indeed, if $x=x_{0}, x_{1}, \ldots, x_{n}=y$ is the shortest traveling salesman tour that starts from $x$, covers $\operatorname{supp} f^{-1} g$, and ends at $y$, then

$$
\begin{aligned}
d((f, x),(g, y)) & \lesssim \sum_{i=0}^{n-1} d_{H}\left(x_{i}, x_{i+1}\right) \leq \sum_{i=0}^{n-1}\left(d_{H}\left(x_{i}, x_{0}\right)+d_{H}\left(x_{i+1}, x_{0}\right)\right) \\
& =d_{H}(x, y)+2 \sum_{z \in \mathbf{s u p p} f^{-1} g} d_{H}(x, z) \\
& \lesssim\left(1+\left|\operatorname{supp} f^{-1} g\right|\right) \cdot \max \left\{d_{H}(x, y) \cup\left\{d_{H}(x, z)\right\}_{z \in \operatorname{supp} f^{-1} g}\right\}
\end{aligned}
$$

Taking $\varepsilon$ to 0 finishes the proof.

Proof of Theorem 1.1. Let $\alpha=\alpha_{1}^{*}(H)$ and $f: H \rightarrow L_{1}$ be a Lipschitz map with compression exponent $\alpha-\varepsilon$ for some $\varepsilon>0$. By the proposition, we then have a Lipschitz map $\Psi: \mathcal{L}_{G}(H) \rightarrow L_{1}$ with compression exponent $\frac{\alpha-\varepsilon}{1+\alpha-\varepsilon}$. Thus, we have proven that $\alpha_{1}^{*}\left(\mathcal{L}_{G}(H)\right) \geq \frac{\alpha_{1}^{*}(H)}{1+\alpha_{1}^{*}(H)}$. Note that as $\alpha_{1}^{*}\left(\mathcal{L}_{G}(H)\right) \leq 1$ always, Theorem 3.3 of $\left[\mathrm{NP} 08\right.$ gives that $\alpha_{1}^{*}(G \succ H) \geq \min \left\{\alpha_{1}^{*}(G), \frac{\alpha_{1}^{*}(H)}{1+\alpha_{1}^{*}(H)}\right\}$.

The case $p>1$ in Theorem 1.1 is a consequence of the general fact that $\alpha_{p}^{*}(\Gamma) \geq$ $\max \left\{\frac{1}{p}, \frac{1}{2}\right\} \alpha_{1}^{*}(\Gamma)$, which holds for any finitely generated group $\Gamma$. This simple fact is explained in [NP09] (see the paragraph following question 10.3 there for a more general statement). For completeness we will now recall how this is proved. As there is a map $T_{p}: L_{1} \rightarrow L_{p}$ such that $\left\|T_{p}(x)-T_{p}(y)\right\|_{p}^{p}=\|x-y\|_{1}$ [Mat02, WW75], we can compose the embedding of $\Gamma$ into $L_{1}$ with $T_{p}$ to get the result that 
$\alpha_{p}^{*}(\Gamma) \geq \frac{1}{p} \alpha_{1}^{*}(\Gamma)$. For $p>2$, we can first embed into $L_{2}$ and then use the fact that $L_{2}$ embeds isometrically into $L_{p}$ for all $p$ (see e.g. Woj91, BL00) to get the final bound $\alpha_{p}^{*}(\Gamma) \geq \max \left\{\frac{1}{2}, \frac{1}{p}\right\} \alpha_{1}^{*}(\Gamma)$. Applying this to our lower bound for $\alpha_{1}^{*}(G \nmid H)$ gives the proof of Theorem 1.1.

\section{CoArse Embeddability}

We will prove only the $L_{1}$ embedding case as the general $L_{p}$ case follows from the second half of the proof of Theorem 1.1. Note that as word metrics take only integer values, we may view the bounding functions as unbounded increasing functions from $\mathbb{Z}_{+}$to $\mathbb{R}_{+}$. We can assume 0 is mapped to 0 .

We begin by proving a quantitative analogue of a lemma from [NP08. The proof will be mostly identical to the original version with a few key changes.

Lemma 3.1. If $G$ and $\mathcal{L}_{G}(H)$ both coarsely embed into $L_{1}$, then $G$ ? $H$ coarsely embeds into $L_{1}$.

Proof. Let $\ell_{1}(H, G$; fin) denote the metric space of all finitely supported functions $f: H \rightarrow G$ equipped with the metric

$$
d_{\ell_{1}}(f, g)=\sum_{z \in H} d_{G}(f(z), g(z))
$$

Then one sees that

$$
d_{G l H}((f, x),(g, y)) \asymp d_{\mathcal{L}_{G}(H)}((f, x),(g, y))+d_{\ell_{1}}(f, g) .
$$

Indeed, we may suppose $(g, y)=(\mathbf{e}, e)$ as the metrics are $G \imath H$-invariant. Then to move from $(\mathbf{e}, e)$ to $(f, x)$ is the same as visiting all locations of $\operatorname{supp} f$ and at each location moving $G$ from $e$ to $f(z) \in G$.

Let $\phi: G \rightarrow L_{1}$ and $\Psi: \mathcal{L}_{G}(H) \rightarrow L_{1}$ be coarse embeddings with the bounds

$$
\begin{aligned}
\tau_{1}(d(x, y)) & \leq\|\phi(x)-\phi(y)\|_{1} \leq \tau_{2}(d(x, y)), \\
\xi_{1}\left(d_{\mathcal{L}_{G}(H)}((f, x),(g, y))\right) & \leq\|\Psi(f, x)-\Psi(g, y)\|_{1} \leq \xi_{2}\left(d_{\mathcal{L}_{G}(H)}((f, x),(g, y))\right) .
\end{aligned}
$$

Define the function $F: G \imath H \rightarrow L_{1} \oplus \ell_{1}\left(H, L_{1}\right.$; fin $)$ by

$$
F(f, x)=\Psi(f, x) \oplus(\phi \circ f) .
$$

We have that $d_{G \zeta H}((f, x),(g, y)) \asymp d_{\mathcal{L}_{G}(H)}((f, x),(g, y))+d_{\ell_{1}(H, G)}(f, g)$,

$$
\begin{aligned}
\|F(f, x)-F(g, y)\|_{1} & =\|\Psi(f, x)-\Psi(g, y)\|_{1}+\sum_{z \in H}\|\phi(f(z))-\phi(g(z))\|_{1} \\
& \leq \xi_{2}\left(d_{\mathcal{L}_{G}(H)}((f, x),(g, y))\right)+\sum_{z \in H} \tau_{2}(d(f(z), g(z))) \\
& \leq \eta_{2}\left(d_{\mathcal{L}_{G}(H)}((f, x),(g, y))+\sum_{z \in H} d(f(z), g(z))\right) \\
& \lesssim \eta_{2}\left(d_{G(H}((f, x),(g, y))\right),
\end{aligned}
$$


where $\eta_{2}(t):=t \cdot\left(\xi_{2}(t)+\tau_{2}(t)\right)$. As $\eta_{2}$ is clearly unbounded increasing, we have an upper bound. Bounding from below, we have

$$
\begin{aligned}
\|F(f, x)-F(g, y)\|_{1} & \geq \xi_{1}\left(d_{\mathcal{L}_{G}(H)}((f, x),(g, y))\right)+\sum_{z \in H} \tau_{1}(d(f(z), g(z))) \\
& \geq \eta_{1}\left(d_{\mathcal{L}_{G}(H)}((f, x),(g, y))+\sum_{z \in H} d(f(z), g(z))\right) \\
& \geq \eta_{1}\left(d_{G \zeta H}((f, x),(g, y))\right),
\end{aligned}
$$

where

$$
\eta_{1}(t):=\min \left\{\xi_{1}\left(t_{1}\right)+\sum_{i=2}^{n} \tau_{1}\left(t_{i}\right): t_{i} \geq 0, \sum_{i=1}^{n} t_{i}=t\right\} .
$$

One can think of this function as evaluated on integer partitions of $t$. To show that $\eta_{1}$ is increasing, take a partition of $t+1$. If all the elements in the partition are of size 1 , then the value would be $\xi_{1}(1)+\sum_{i=1}^{t} \tau_{1}(1)$, which is greater than the value for the partition of $t$ of all sizes 1 as $\xi_{1}(1)>0$ and $\tau_{1}(1)>0$. If the partition $t+1$ has an element of value greater than 1 , then reducing this by one gives a partition of $t$ of lesser value as $\xi_{1}$ and $\tau_{1}$ are increasing. Thus, we see that the minimum of the partitions of $t+1$ is always greater than the minimum of the partitions of $t$. It remains to show that $\eta_{1}$ is unbounded.

Let $M>0$. By rescaling, we may suppose that $\xi_{1}(1) \geq 1$ and $\tau_{1}(1) \geq 1$. Let $N>0$ such that $\tau_{1}(N) \geq M$ and $\xi_{1}(N) \geq M$. Consider the possible partitions of $M N$. If there are more than $M$ elements of the partition, then as $\tau_{1}(1) \geq 1$ and $\xi_{1}(1) \geq 1$, the summation associated to this partition would have value greater than $M$. Thus, the number of elements of the partition has to be less than $M$. However, one of elements in the partitions has to have value greater than $N$ by the pigeonhole principle, and so either of the $\xi_{1}$ or $\tau_{1}$ value of this element is greater than $M$. Thus, $\eta_{1}(M N)>M$ and so $\eta_{1}$ is unbounded.

Note that as $\xi_{2}$ and $\tau_{2}$ are increasing unbounded functions, $\eta_{2}$ grows superlinearly. However, as $F$ is Lipschitz, we can always use the triangle inequality to give a linear upper bound based on the expansion between generators. Thus, we may replace $t \cdot\left(\zeta_{2}(t)+\tau_{2}(t)\right)$ with a linear compression bound.

Proof of Theorem 1.2. By the preceding remarks and lemma, it suffices to show that $\mathcal{L}_{G}(H)$ embeds coarsely into $L_{1}$ when $G$ and $H$ embed coarsely into $L_{1}$. As such, let $\psi: H \rightarrow L_{1}$ be an embedding with bounds

$$
\rho_{1}(d(x, y)) \leq\|\psi(x)-\psi(y)\|_{1} \leq \rho_{2}(d(x, y)) .
$$

Using the cut decomposition, we construct the function $F: \mathcal{L}_{G}(H) \rightarrow L_{1}$ from the measure $\nu$ on cuts of $\mathcal{L}_{G}(H)$ as before. As before, we have that

$$
\|F(f, x)-F(g, y)\|_{1} \asymp \mu\left(\left\{B \in \mathcal{C}: B \vdash\left(\operatorname{supp} f^{-1} g\right) \cup\{x, y\}\right\}\right) .
$$

In particular,

$$
\|F(f, x)-F(g, y)\|_{1} \gtrsim \max \left\{\rho_{1}(d(x, y)) \cup\left\{\rho_{1}(d(x, z))_{z \in\left(\operatorname{supp} f^{-1} g\right) \cup\{y\}}\right\}\right\}
$$


and

$$
\begin{aligned}
\|F(f, x)-F(g, y)\|_{1} & \lesssim \mu\left(\left\{B \in \mathcal{C}: B \vdash\left(\operatorname{supp} f^{-1} g\right) \cup\{x, y\}\right\}\right) \\
& \leq \sum_{u, v \in\left(\operatorname{supp} f^{-1} g\right) \cup\{x, y\}}\|\psi(u)-\psi(v)\|_{1} \\
& \leq \sum_{u, v} \rho_{2}(d(u, v)) .
\end{aligned}
$$

Construct the mapping $\Psi(f, x):=\Lambda(f) \oplus F(f, x)$. Bounding from above, we have that

$$
\begin{aligned}
\|\Psi(f, x)-\Psi(g, y)\|_{1} & \lesssim\left|\operatorname{supp} f^{-1} g\right|+\sum_{u, v \in\left(\mathbf{s u p p} f^{-1} g\right) \cup\{x, y\}} \rho_{2}(d(u, v)) \\
& \leq d((f, x),(g, y))+\sum_{u, v} \rho_{2}(d((f, x),(g, y))) \\
& \leq \tau_{2}(d((f, x),(g, y))),
\end{aligned}
$$

where $\tau_{2}(t):=t+t^{2} \cdot \rho_{2}(t)$. Bounding from below, we have

$$
\begin{aligned}
\| \Psi & (f, x)-\Psi(g, y) \|_{1} \\
& \gtrsim\left|\operatorname{supp} f^{-1} g\right|+\max \left\{\rho_{1}(d(x, y)) \cup\left\{\rho_{1}(d(x, z))_{z \in\left(\operatorname{supp} f^{-1} g\right) \cup\{y\}}\right\}\right\} \\
& \gtrsim \max \left\{\sqrt{\left|\operatorname{supp} f^{-1} g\right| \cdot \max \left\{\rho_{1}(d(x, y)) \cup\left\{\rho_{1}(d(x, z))_{z \in\left(\operatorname{supp} f^{-1} g\right) \cup\{y\}}\right\}\right.}, \rho_{1}(d(x, y))\right\} \\
& \gtrsim \sqrt{\left(1+\left|\operatorname{supp} f^{-1} g\right|\right) \cdot \max \left\{\rho_{1}(d(x, y)) \cup\left\{\rho_{1}(d(x, z))_{z \in\left(\operatorname{supp} f^{-1} g\right) \cup\{y\}}\right\}\right.} \\
& \geq \tau_{1}(d((f, x),(g, y))),
\end{aligned}
$$

where

$$
\tau_{1}(t):=\sqrt{\min \left\{\sum_{k=1}^{n} \rho_{1}\left(s_{k}\right): s_{k} \geq 0, \sum_{k=1}^{n} s_{k}=s\right\}} .
$$

The second inequality came from using the AM-GM inequality. We can also use Young's inequality rather than the AM-GM inequality to improve the lower bound for certain lower moduli. Using a similar proof as above, we can see that $\tau_{1}$ is an increasing unbounded function. Thus, we have that $\mathcal{L}_{G}(H)$ embeds coarsely into $L_{1}$. Composing these compression bounds with those of Lemma 2.1 gives us the necessary bounds.

\section{The HaAgerup property}

In this section, we use the ideas from above to show that equivariant maps into Hilbert space can be amalgamated to give an equivariant map defined on $G$ ? $H$. As before we first prove the $L_{1}$ analogue.

Theorem 4.1. If $G$ and $H$ admit metrically proper equivariant mappings into $L_{1}$, then so does $G$ ? $H$.

As above, we need to prove the following lemma

Lemma 4.1. If $G$ and $\mathcal{L}_{G}(H)$ admit equivariant mappings into $L_{1}$, then so does $G$ 々 $H$. 
Proof. Let $\psi: G \rightarrow L_{1}$ and $\phi: \mathcal{L}_{G}(H) \rightarrow L_{1}$ be equivariant maps with associated actions $\tau$ and $\pi$. We would like to show that

$$
\begin{aligned}
\Psi: G \curlywedge H & \rightarrow L_{1} \oplus \ell_{1}\left(H, L_{1} ; \text { fin }\right) \\
(f, x) & \mapsto \phi(f, x) \oplus(\psi \circ f)
\end{aligned}
$$

is equivariant. We will express elements of $\ell_{1}(H, X$; fin) as elements of the direct product $\bigoplus_{h \in H} X$. The action of $H$ on this direct product is then permutation of coordinates. This is precisely the action of $T_{h}$ for $h \in H$. Note that the semidirect product of $\bigoplus_{h \in H} G_{h}$ with $H$ by this action is just the wreath product $G$ 々 H. Consider the group action of $G \imath H$ on $L_{1} \oplus \ell_{1}\left(H, L_{1}\right.$; fin),

$$
\theta(f, x)\left(u, \sum_{h \in H} g_{h}\right)=\left(\pi(f, x) u, \sum_{h \in H} \tau(f(h)) g_{x^{-1} h}\right),
$$

where $g_{h} \in L_{1}$. It is then straightforward from the formulas to see that

$$
\Psi((f, x) \cdot(g, y))=\theta(f, x) \cdot \Psi(g, y)+\Psi(f, x) .
$$

We also require the following theorem Lam58, (see also the exposition in [FJ03]).

Theorem 4.2 (Lamperti's Theorem). Let $U$ be an isometry of $L_{1}$ onto itself. Then there is a Borel measurable self-mapping $\varphi$ of $[0,1]$ that is bijective almost everywhere and $a u \in L_{1}$ such that

$$
U \psi=u \cdot(\psi \circ \varphi) .
$$

Furthermore,

$$
\int_{\varphi^{-1}(E)}|u| d t=\int_{E} d t
$$

for every Borel set E.

As $U$ is an isometry, it is clear that $\varphi$ cannot map a set of positive measure to a set of measure 0 and vice versa. In addition, $u$ must be nonzero on a set of full measure.

Proof of Theorem 4.1. By the preceding lemma, it suffices to show that the map $F$ on $\mathcal{L}_{G}(H)$ constructed as before from $\psi: H \rightarrow L_{1}$ is equivariant. Indeed, the mapping $(f, x) \mapsto \Lambda(f)$ is equivariant and so the entire embedding $\Psi(f, x)=$ $F \oplus \Lambda(f)$ would be equivariant. As we are using the same construction of $\Psi$ as before, that $\Psi$ is metrically proper will follow from the arguments of Section 3 .

Recall that the cuts generated in the decomposition of $\psi$ are given by the cut map

$$
\begin{aligned}
S:[0,1] \times \mathbb{R} & \rightarrow \mathcal{C} \\
(y, t) & \mapsto\left\{h \in H: t^{-1} \cdot \psi(h)(y)>1\right\},
\end{aligned}
$$

and the measure $\rho$ is the pushforward of the Lebesgue measure on $[0,1] \times \mathbb{R}$ by $S$. As before, we complete $\mathcal{C}$ under taking complements and define a new complementinvariant measure $\mu$ on $\mathcal{C}$ from $\rho$. Having defined $\mu$, we extend it to $\nu$ on $\mathcal{H}$ as before and from this we get a map $F: \mathcal{L}_{G}(H) \rightarrow L_{1}(\mathcal{H}, \nu)$ with the desired properties.

We would like to show that there exists an isometric group action of $\mathcal{L}_{G}(H)$ on $L_{1}(\mathcal{H}, \nu)$ such that $F(g h)=\pi(g) \cdot F(h)+F(g)$. We accomplish this by showing 
that the natural action of $H$ on the set of cuts $\mathcal{C}$ by left multiplication is measurepreserving. Note that we can extend the action of $H$ to $\mathcal{H}$ by

$$
h E_{i}(B, f)=E_{i}\left(h B, T_{h} f\right) .
$$

The problem comes from determining whether $h B$ is in $\mathcal{C}$. Given a finitely supported function $g: H \rightarrow G$, we can also specify the action

$$
g E_{i}(B, f)=E_{i}\left(B,\left.g\right|_{B^{c}} \cdot f\right) .
$$

These two actions are easily seen to be compatible with the group operation. Thus, to show that this is actually a group action, it suffices to show that $\mathcal{C}$ is $H$-invariant except possibly on a set of measure 0 . To show that this group action is isometric, we require that $\nu$ be $G$ ? $H$-invariant. By the pullback construction of $\nu$, it suffices to show that $\rho$ is $H$-invariant.

Let $\varphi$ and $u$ be the functions associated to $\pi(h)$ by Lamperti's theorem. From equivariance, we have that

$$
\begin{aligned}
\psi(h g)\left(\varphi^{-1}(y)\right) & =\pi(h) \cdot \psi(g)\left(\varphi^{-1}(y)\right)+\psi(h)\left(\varphi^{-1}(y)\right) \\
& =u\left(\varphi^{-1}(y)\right) \cdot \psi(g)(y)+\psi(h)\left(\varphi^{-1}(y)\right)
\end{aligned}
$$

for almost every $y \in[0,1]$. As we only need to prove $H$-invariance on a full measure subset of $\mathcal{C}$, we may suppose that $x=\varphi^{-1}(y)$ is defined and $a=u(x)$ and $b=$ $\psi(h)(x)$ are finite. Suppose $t>0$. If $a \cdot(a t+b)>0$, we have that $h S(y, t)=$ $S(x, a t+b)$. If $a \cdot(a t+b)<0$, then $h S(y, t)=S(x, a t+b)^{c}$. As we required that $\mathcal{C}$ be closed under complement, this is not a problem. The case when $t<0$ can also be similarly analyzed. As $\varphi$ cannot take measure 0 sets onto sets of positive measure and vice versa, this shows that the set of cuts is $H$-invariant up to a set of cuts of measure 0 . It remains to show that $\rho$ is $H$-invariant.

As before, fix $h \in H$ and let $\varphi, u$ be the functions associated to the isometry $\pi(h)$. Let $B \in \mathcal{C}$. Note by the cut map that the quantity $\mu(\{B\})=\rho(\{B\})+\rho\left(\left\{B^{c}\right\}\right)$ can be thought of as the area bound between the two families of curves $\{\psi(g): g \in B\}$ and $\left\{\psi(g): g \in B^{c}\right\}$, that is, the Lebesgue measure of the points of $[0,1] \times \mathbb{R}$ that is below all the graphs of one family and above all the graphs of the other.

Note that $\pi(h)$ induces a self-mapping of $[0,1] \times \mathbb{R}$. Indeed, taking the arguments of the $H$-invariance into account, the transformation can be given by

$$
\pi(h)(x, y)=\left(\varphi^{-1}(x), y \cdot u\left(\varphi^{-1}(x)\right)+\psi(h)\left(\varphi^{-1}(x)\right)\right) .
$$

By Lamperti's theorem, this transformation, which is defined on a set of full measure, is precisely the one that takes the graph of $\psi(g)$ to the graph of $\psi(h g)$. Note that vertical ordering of the graphs is preserved by this transformation at each $x \in[0,1]$ except with the possibility of a flip. Let $E \times F \subset[0,1] \times \mathbb{R}$ be a measurable subset,

$$
\int_{\pi(h)(E \times F)} d x d y=\int_{\varphi^{-1}(E)} \int_{F}|u(x)| d y d x=|F| \int_{\varphi^{-1}(E)}|u| d x=|E||F| .
$$

Thus, we see that $\pi(h)$ is measure-preserving for the set of generators of the $\sigma$-algebra of $[0,1] \times \mathbb{R}$. As area is preserved, we have that $\mu(h B)=\mu(B)$. It follows that $H$ induces a measure-preserving transformation on $(\mathcal{H}, \nu)$ and subsequently, an isometry of $L_{1}(\mathcal{H}, \nu)$, which we will still denote $\pi$.

Note that the cut map $S:[0,1] \times \mathbb{R} \rightarrow(\mathcal{C}, \mu)$ induces an isometric embedding of $L_{1}(\mathcal{C}, \mu)$ into $L_{1}([0,1] \times \mathbb{R})$. It is readily seen that $L_{1}(\tilde{C}, \tilde{\mu})$ is a countable $\ell_{1}$ 
sum of $L_{1}(\mathcal{C}, \mu)$ and so isometrically embeds into $\left(\sum_{j=1}^{\infty} L_{1}([0,1] \times \mathbb{R})\right)_{1}$, which is isometric to $L_{1}$. Note that $\iota$, the injection of $\mathcal{H}$ into $\tilde{\mathcal{C}}$, has the property that $\nu\left(\iota^{-1}(E)\right) \leq \tilde{\mu}(E)$. This gives that the induced map $\iota^{*}: L_{1}(\tilde{\mathcal{C}}, \tilde{\mu}) \rightarrow L_{1}(\mathcal{H}, \nu)$ is continuous and onto and so $L_{1}(\mathcal{H}, \nu)$ is separable.

As $L_{1}(\mathcal{H}, \nu)$ is separable, we know that it is isometric to one of the following spaces Woj91:

$$
L_{1}, \ell_{1},\left\{\ell_{1}^{n}\right\}_{n=1}^{\infty}, L_{1} \oplus \ell_{1},\left\{L_{1} \oplus \ell_{1}^{n}\right\}_{n=1}^{\infty} .
$$

If $L_{1}(\mathcal{H}, \nu)$ is isometric to $L_{1}$, then we are done. Otherwise, we need to embed $L_{1}(\mathcal{H}, \nu)$ into $L_{1}$ and define a suitable isometric action. The argument will follow closely to the ones made in NP09.

If $L_{1}(\mathcal{H}, \mu)$ is isometric to $\ell_{1}$, then Lamperti's theorem tells us that $\pi(g) e_{i}=$ $\theta_{i}^{g} e_{\tau^{g}(i)}$ for all $i \in \mathbb{N}$, where $\left\{e_{i}\right\}_{i=1}^{\infty}$ is the standard coordinate basis for $\ell_{1}$, the function $\tau^{g}: \mathbb{N} \rightarrow \mathbb{N}$ is bijective, and $\left|\theta_{i}^{g}\right| \equiv 1$. Embedding $L_{1}(\mathcal{H}, \nu)$ into $L_{1}$ by the standard mapping $\varphi: x \mapsto \sum_{i=1}^{\infty} 2^{i} x_{i} \chi_{\left[2^{-i}, 2^{-i+1}\right]}$, we can define the isometric action of $H$ on $L_{1}$, which we will still denote by $\pi$, by

$$
\pi(g) f(t):=\theta_{i}^{g} f\left(2^{i-\tau^{g}(i)} t\right) .
$$

It is immediate to check that $\pi$ and $\varphi \circ f$ satisfy the necessary equivariance relation.

For the cases when $L_{1}(\mathcal{H}, \nu)$ is isometric to $L_{1} \oplus \ell_{1}(S)$, where $S$ is a countable set, we use Lamperti's theorem to show that isometric automorphisms map disjoint functions to disjoint functions and indicators of atoms to indicators of atoms. Thus, $\pi(g)$ restricts to isometries of each summand. By embedding $L_{1} \oplus \ell_{1}(S)$ into $L_{1} \oplus L_{1}$ (which is isometric to $L_{1}$ ), we can get the necessary isometric action by using the results from above on each of the $L_{1}$ summands.

Proof of Theorem 1.3. We will use the same embedding as above except for a change in the first step of embedding $L_{2}$ into $L_{1}$. Given an equivariant map $\psi: H \rightarrow L_{2}$, the proof of Lemma 2.3 in [NP08] shows that there exists an equivariant map $\tilde{\psi}: H \rightarrow L_{1}$ such that $\|\tilde{\psi}(x)-\tilde{\psi}(y)\|_{1}=C \cdot\|\psi(x)-\psi(y)\|_{2}$ for some constant $C$.

In conjunction with the previous theorem, we have that the existence of an equivariant map $f: H \rightarrow L_{2}$ gives an equivariant map $\left.F: G\right\} H \rightarrow L_{1}$. Let $\pi$ be the associated group action on $L_{1}$. It remains to show that the map $T \circ F: G$ ? $H \rightarrow L_{2}$ is also equivariant. Recall that the embedding $T$ of $L_{1}[0,1]$ into $L_{2}([0,1] \times \mathbb{R})$ can be thought of as a mapping $f$ to $(x, y) \mapsto 1-\mathbf{1}_{[0, y]}(f(x))$. This maps a function on $[0,1]$ to the characteristic function on the area bound by its graph. As above, we may think of $\pi(f, x)$ as a measure-preserving transformation of $[0,1] \times \mathbb{R}$ given by

$$
\pi(f, x)(y, t)=\left(\varphi^{-1}(y), t \cdot u\left(\varphi^{-1}(y)\right)+F(f, x)\left(\varphi^{-1}(y)\right)\right) .
$$

This induces an isometry of $L_{2}([0,1] \times \mathbb{R})$. Given $\pi(f, x)$ and $\pi(g, y)$ with corresponding functions, $u_{1}, \varphi_{1}$ and $u_{2}, \varphi_{2}$, we see that

$$
\pi((f, x)(g, y)) \cdot \psi(t)=\pi(f, x)\left(u_{2}(t) \cdot \psi\left(\varphi_{2}(t)\right)\right)=u_{1}(t) \cdot u_{2}\left(\varphi_{1}(t)\right) \cdot \psi\left(\varphi_{2} \circ \varphi_{1}(t)\right) .
$$

Thus, the associated functions for $\pi((f, x) \cdot(g, y))$ are $u_{1}(t) \cdot u_{2}\left(\varphi_{1}(t)\right)$ and $\varphi_{2} \circ \varphi_{1}$. The first coordinate of the mapping clearly preserves the group structure. For the second coordinate, using the fact that $F$ is equivariant with respect to the action 
$\pi$, we get

$$
\begin{aligned}
\pi(f, x) \pi(g, y) \cdot(y, t) & =\pi(f, x)\left(\varphi_{2}^{-1}(y), t \cdot u_{2}\left(\varphi_{2}^{-1}(y)\right)+F(g, y)\left(\varphi_{2}^{-1}(y)\right)\right) \\
& =\left(\varphi_{1}^{-1}\left(\varphi_{2}^{-1}(y)\right), t \cdot u_{2}\left(\varphi_{2}^{-1}(y)\right) u_{1}\left(\varphi_{1}^{-1}\left(\varphi_{2}^{-1}(y)\right)\right)\right. \\
& \left.+u_{1}\left(\varphi_{1}^{-1}\left(\varphi_{2}^{-1}(y)\right)\right) F(g, y)\left(\varphi_{2}^{-1}(y)\right)+F(f, x)\left(y^{\prime}\right)\right) \\
& =\left(y^{\prime}, t \cdot u_{2}\left(\varphi_{1}\left(y^{\prime}\right)\right) u_{1}\left(y^{\prime}\right)+F((f, x)(g, y))\left(y^{\prime}\right)\right) \\
& =\pi((f, x)(g, y))(y, t) .
\end{aligned}
$$

Thus, $\pi$ is indeed a group action. By the same arguments as above, this is a measure-preserving group action and so induces a group action of $L_{2}([0,1] \times \mathbb{R})$, which we denote by $\tau$. One can see from the definition of $T$ then that $T \circ F((f, x)$. $(g, y))=\tau(f, x) \cdot(T \circ F(g, y))+T \circ F(f, x)$.

\section{ACKNOWLEDGEMENT}

I am grateful to Assaf Naor for introducing me to the problems studied here and for suggesting that I look at dCSV09 in this context. I also thank him for his improvement of my initial result, which was $\alpha_{1}^{*}(G \succ H) \geq \min \left\{\alpha_{1}^{*}(G), \frac{\alpha_{1}^{*}(H)}{2}\right\}$. Note that, using the notation of Remark 1.1. this weaker bound would yield the bound $\alpha_{1}^{*}\left(G_{k}\right) \geq \frac{1}{2^{k-1}}$ rather than our result $\alpha_{1}^{*}\left(G_{k}\right) \geq \frac{1}{k}$, though, as asked in Remark 1.1, it might be the case that there is actually a universal lower bound on $\alpha_{1}^{*}\left(G_{k}\right)$. Lastly, I am grateful to Assaf Naor for many constructive discussions and for his many revisions of this manuscript.

\section{REFERENCES}

[AGS06] G.N. Arzhantseva, V.S. Guba, and M.V. Sapir, Metrics on diagram groups and uniform embeddings in a Hilbert space, Comment. Math. Helv. 81 (2006), no. 4, 911-929. MR.2271228 (2007k:20084)

[ANP09] T. Austin, A. Naor, and Y. Peres, The wreath product of $\mathbb{Z}$ with $\mathbb{Z}$ has Hilbert compression exponent $\frac{2}{3}$, Proc. Amer. Math. Soc. 137 (2009), no. 1, 85-90. MR 2439428 (2009f:20060)

[BL00] Y. Benyamini and J. Lindenstrauss, Geometric nonlinear functional analysis. Vol. 1, American Mathematical Society Colloquium Publications, vol. 48, American Mathematical Society, 2000. MR 1727673 (2001b:46001)

$\left[\mathrm{CCJ}^{+} 01\right]$ P.-A. Cherix, M. Cowling, P. Jolissaint, P. Julg, and A. Valette, Groups with the Haagerup property, Progress in Mathematics, vol. 197, Birkhäuser Verlag, 2001. MR:1852148 (2002h:22007)

[CK06] J. Cheeger and B. Kleiner, Differentiating maps into $L^{1}$ and the geometry of $B V$ functions, to appear in Ann. of Math.

[dCSV09] Y. de Cornulier, Y. Stalder, and A. Valette, Proper actions of wreath products and generalizations, preprint, 2009.

[DL97] M.M. Deza and M. Laurent, Geometry of cuts and metrics, Algorithms and Combinatorics, vol. 15, Springer-Verlag, 1997. MR1460488 (98g:52001)

[FJ03] R.J. Fleming and J.E. Jamison, Isometries on Banach spaces: function spaces, Chapman \& Hall/CRC Monographs and Surveys in Pure and Applied Mathematics, vol. 129, Chapman \& Hall/CRC, 2003. MR1957004 (2004j:46030)

[Gal08] Ś.R. Gal, Asymptotic dimension and uniform embeddings, Groups Geom. Dyn. 2 (2008), no. 1, 63-84. MR2367208 (2009d:20105)

[GK04] E. Guentner and J. Kaminker, Exactness and uniform embeddability of discrete groups, J. London Math. Soc. (2) 70 (2004), no. 3, 703-718. MR2160829(2006i:43006)

[Lam58] J. Lamperti, On the isometries of certain function spaces, Pacific J. Math. 8 (1958), 459-466. MR0105017 (21:3764) 
[Mat02] J. Matoušek, Lectures on discrete geometry, Graduate Texts in Mathematics, vol. 212, Springer-Verlag, 2002. MR.1899299 (2003f:52011)

[NP08] A. Naor and Y. Peres, Embeddings of discrete groups and the speed of random walks, Int. Math. Res. Not. (2008). MR2439557 (2009m:20067)

[NP09] $\longrightarrow L_{p}$ compression, traveling salesmen, and stable walks, preprint.

[SV07] Y. Stalder and A. Valette, Wreath products with the integers, proper actions and Hilbert space compression, Geom. Dedicata 124 (2007), 199-211. MR2318545 (2008i:20053)

[Tes09] R. Tessera, Asymptotic isoperimetry on groups and uniform embeddings into Banach spaces, to appear in Comment. Math. Helv., 2009.

[Woj91] P. Wojtaszczyk, Banach spaces for analysts, Cambridge Studies in Advanced Mathematics, vol. 25, Cambridge University Press, 1991. MR.1144277 (93d:46001)

[WW75] J.H. Wells and L.R. Williams, Embeddings and extensions in analysis, Springer-Verlag, 1975. MR0461107 (57:1092)

Courant Institute of Mathematical Sciences, 251 Mercer Street, New York, NEW YORK 10012-1185

E-mail address: seanli@cims.nyu.edu 